\title{
Noise robustness of the nonlocality of entangled quantum states
}

\author{
Mafalda L. Almeida ${ }^{1}$, Stefano Pironio ${ }^{1}$, Jonathan Barrett ${ }^{2}$, Géza Tóth ${ }^{1,3}$, and Antonio Acín ${ }^{1,4}$ \\ ${ }^{1}$ ICFO-Institut de Ciencies Fotoniques, E-08860 Castelldefels, Barcelona, Spain \\ ${ }^{2}$ Perimeter Institute for Theoretical Physics, 31 Caroline Street N, Waterloo, Ontario, Canada N2L $2 Y 5$ \\ ${ }^{3}$ Research Institute for Solid State Physics and Optics, P.O. Box 49, H-1525 Budapest, Hungary \\ ${ }^{4}$ ICREA-Institució Catalana de Recerca i Estudis Avançats, Lluis Companys 23, 08010 Barcelona, Spain
}

(Dated: October 26, 2018)

\begin{abstract}
We study the nonlocal properties of states resulting from the mixture of an arbitrary entangled state $\rho$ of two $d$-dimensional systems and completely depolarized noise, with respective weights $p$ and $1-p$. We first construct a local model for the case in which $\rho$ is maximally entangled and $p$ at or below a certain bound. We then extend the model to arbitrary $\rho$. Our results provide bounds on the resistance to noise of the nonlocal correlations of entangled states. For projective measurements, the critical value of the noise parameter $p$ for which the state becomes local is at least asymptotically $\log (d)$ larger than the critical value for separability.
\end{abstract}

In 1964, Bell showed that some entangled states are nonlocal, in the sense that measurements on them yield outcome correlations that cannot be reproduced by a locally causal model [1]. This nonlocal character of entangled states may be demonstrated through the violation of Bell inequalities. All pure entangled states violate such an inequality, hence are nonlocal [2]. For noisy states, the picture is much subtler. Werner constructed in 1989 a family of bipartite mixed states, which, while being entangled, return outcome correlations under projective measurements that can be described by a local model 3 . This result has been extended to general measurements [4] and more parties [5]. Thus, while entanglement is necessary for a state to be nonlocal, in the case of mixed states it is not sufficient.

Beyond these exploratory results, little is known about the relation between noise, entanglement, and quantum nonlocality. Understanding this relation, apart from its fundamental interest, is important from the perspective of Quantum Information Science. In this context, entanglement is commonly viewed as a useful resource for various information-processing tasks. Not all entangled states, however, are useful for every task: for example, quantum computation with slightly entangled states can be efficiently simulated on a classical computer [6], and bound entangled states are useless for teleportation [7]. For certain tasks, such as quantum communication complexity problems [8], or device-independent quantum key distribution [9], entangled states are useful only to the extent that they exhibit nonlocal correlations. Indeed, in these scenarios two (or more) distant observers, Alice and Bob, directly exploit the correlations

$$
P_{M N}(a, b)=\operatorname{tr}\left(\rho_{A B} M_{a} \otimes N_{b}\right),
$$

obtained by performing measurements $M$ and $N$ on a distributed entangled state $\rho_{A B}$ (in the above formula, $M_{a}$ and $N_{b}$ are the positive operators associated with the measurement outcomes $a$ and $b$ ). If the entangled state $\rho_{A B}$ can be simulated by a local model, these correlations can be written as

$$
P_{M N}(a, b)=\int \mu(d \lambda) P_{M}(a \mid \lambda) P_{N}(b \mid \lambda),
$$

where $\lambda$ denotes a shared classical variable distributed with probability measure $\mu$, and $P_{M}(a \mid \lambda)$ and $P_{N}(b \mid \lambda)$ are the local response functions of Alice and Bob. For all practical purposes then, the entangled state $\rho_{A B}$ can be replaced by classical correlations, and so does not provide any improvement over what is achievable using classical resources [10].

In this work, we estimate the resistance to noise of the nonlocal correlations of bipartite entangled states in $\mathbb{C}^{d} \otimes$ $\mathbb{C}^{d}$, where $d$ is the local Hilbert space dimension of each subspace. To do this, we analyze the nonlocal properties of states resulting from the mixture of an arbitrary state $\rho$ with completely depolarized noise,

$$
\rho(p)=p \rho+(1-p) \frac{\mathbb{1}}{d^{2}} .
$$

Our goal is to find the minimal amount of noise that destroys the nonlocal correlations of any state $\rho$, i.e., the maximal value $p_{L}$ such that $\rho(p)$ is local for any $\rho$ when $p \leq p_{L}$. Clearly, for sufficiently small values of $p \leq p_{S}$, the state $\rho(p)$ becomes separable for any $\rho$ [11, 12], thus local. We give here lower bounds on $p_{L}$ that are more constraining than the one obtained from the separability condition. If we restrict Alice and Bob to perform projective measurements only, the bound that we obtain for the locality limit is asymptotically $\log (d)$ larger than the separability limit.

A key step in the proof of our results is the construction of a local model for states of the form (3) when $\rho=\left|\phi_{d}\right\rangle\left\langle\phi_{d}\right|$ is maximally entangled, i.e. $\left|\phi_{d}\right\rangle=$ $1 / \sqrt{d} \sum_{i=1}^{d}|i i\rangle$. Thus we also provide a lower bound on $p_{L}^{\phi}$, defined as the maximal value of $p$ such that

$$
p\left|\phi_{d}\right\rangle\left\langle\phi_{d}\right|+(1-p) \frac{\mathbb{1}}{d^{2}}
$$

is local. This last result implies in particular the existence of entangled states whose nonlocal correlations are 
more robust than those of maximally entangled ones.

The results presented here concern mostly the simpler but physically relevant case in which Alice and Bob are restricted to projective measurements. Extensions to completely general measurements are discussed at the end of the paper. Our results also provide bounds for the notion of state steerability introduced in [13].

As mentioned, we start by analyzing the case in which the state $\rho$ in (3) is maximally entangled. Such states are called isotropic states and are the unique ones invariant under $U \otimes U^{*}$ transformations for all unitary operators $U$ on $\mathbb{C}^{d}[14]$. If Alice and Bob each make on these states a projective measurement, specified by a set of $d$ orthogonal projectors $Q=\left\{Q_{a}\right\}$ for Alice and $R=\left\{R_{b}\right\}$ for Bob, with $a, b=1, \ldots, d$, the resulting joint outcome probabilities are given by

$$
\frac{p}{d} \operatorname{tr}\left(Q_{a}^{T} R_{b}\right)+\frac{1-p}{d^{2}} .
$$

Our first aim is to construct a local model for isotropic states, that is, to write the quantum probabilities (5) in the form (2) for some value of the noise parameter $p$.

Our construction is inspired by the model given in Ref. [3] for Werner states, which are $U \otimes U$ invariant, and which we adapt to the $U \otimes U^{*}$ symmetry of isotropic states. The local classical variables $\lambda$ in our model are taken to be complex $d$-dimensional vectors which we can thus formally identify with $d$-dimensional quantum states $|\lambda\rangle$. The probability measure $\mu$ is the unique measure invariant under all unitary transformations $U$ on $\mathbb{C}^{d}$. In analogy with the quantum formalism, Alice's response function is defined as

$$
P_{Q}(a \mid \lambda)=\left\langle\lambda\left|Q_{a}^{T}\right| \lambda\right\rangle .
$$

Bob's response function is suggested by the perfect correlations of maximally entangled states and taken to be

$$
P_{R}(b \mid \lambda)= \begin{cases}1 & \text { if }\left\langle\lambda\left|R_{b}\right| \lambda\right\rangle=\max _{i}\left\langle\lambda\left|R_{i}\right| \lambda\right\rangle \\ 0 & \text { otherwise } .\end{cases}
$$

It satisfies

$$
P_{U^{\dagger} R U}(b \mid \lambda)=P_{R}(b \mid U \lambda) .
$$

To obtain the joint probabilities predicted by this model, and to compare them with (5), it is necessary to compute the integral (2) for our specific choice of measure $\mu$ and response functions. Following Werner (see [3] for details), one can show that the $U$-invariance of $\mu$, the form (6) of Alice's response function, and the relation (8) satisfied by Bob's response function, imply

$$
\int \mu(d \lambda) P_{Q}(a \mid \lambda) P_{R}(b \mid \lambda)=\operatorname{tr}\left(Q_{a}^{T} \hat{B}(b, R)\right),
$$

where $\hat{B}(b, R)$ is a positive operator depending on Bob's response function. One can further show, exploiting the fact that the relation (9) holds for all one-dimensional projectors $Q_{a}$ [3], that $\hat{B}(b, R)=\left(p^{\phi} / d\right) R_{b}+(1-$ $\left.p^{\phi}\right) / d^{2} \mathbb{1}$, for some $p^{\phi} \in \mathbb{R}$, and thus that

$$
\int \mu(d \lambda) P_{Q}(a \mid \lambda) P_{R}(b \mid \lambda)=\frac{p^{\phi}}{d} \operatorname{tr}\left(Q_{a}^{T} R_{b}\right)+\frac{1-p^{\phi}}{d^{2}} .
$$

These correlations are thus already of the prescribed form (51). To determine the value of $p^{\phi}$ for which (10) holds, it is sufficient to compute the integral (9) in the simplest case where $Q_{a}^{T}=R_{b}$, which gives

$$
p^{\phi}=\frac{1}{d-1}\left(-1+d^{2} \int \mu(d \lambda)\left\langle\lambda\left|R_{b}\right| \lambda\right\rangle P_{R}(b \mid \lambda)\right) .
$$

It now remains to evaluate this integral for the specific choice (7) for $P_{R}(b \mid \lambda)$. After patient algebra, one obtains

$$
p^{\phi}=\frac{1}{d-1}\left(-1+\sum_{k=1}^{d} \frac{1}{k}\right) \underset{\text { large } d}{\longrightarrow} \frac{\log (d)}{d} .
$$

For $d=2, p^{\phi}=1 / 2$ is equal to the critical value for two-dimensional Werner states, as expected since Werner and isotropic states are equivalent up to local unitary transformations when $d=2$. In the limit of large $d, p^{\phi}$ is asymptotically $\log (d)$ larger than the critical probability $p_{S}^{\phi}=1 /(d+1)$ for the separability of isotropic states [14].

Our next goal is to generalize the local model for isotropic states to mixed states of the form

$$
\rho=p|\psi\rangle\langle\psi|+(1-p) \frac{\mathbb{1}}{d^{2}},
$$

where $|\psi\rangle$ is an arbitrary pure state in $\mathbb{C}^{d} \otimes \mathbb{C}^{d}$. This automatically also implies a model for the general states (3), since any mixed state $\rho$ is a convex combination of pure states. To do this, we incorporate Nielsen's protocol [15] for the conversion of bipartite pure states by local operations and classical communication (LOCC) into our model. Recall that a maximally entangled state $\left|\phi_{d}\right\rangle$ can be transformed by LOCC in a deterministic way into an arbitrary state $|\psi\rangle$ by a single measurement on Alice's particle followed by a unitary operation on Bob's side, depending on Alice's measurement outcome. Indeed, consider an arbitrary pure entangled state written in its Schmidt form $|\psi\rangle=\sum_{j=0}^{d-1} \nu_{j}|j j\rangle$, and denote by $D_{\nu}$ the $d \times d$ diagonal matrix with entries $\left(D_{\nu}\right)_{j j}=\nu_{j}$. Taking the $d$ cyclic permutations $\Pi_{i}=\sum_{j=0}^{d-1}|j\rangle\langle j+i(\bmod d)|$, where $i=0, \ldots, d-1$, it is possible to write

$$
|\psi\rangle=\sqrt{d}\left(A_{i} \otimes \Pi_{i}\right)\left|\phi_{d}\right\rangle \quad \text { for all } i=0, \ldots, d-1,
$$

with $A_{i}=D_{\nu} \Pi_{i}$. The operators $W_{i}=A_{i}^{\dagger} A_{i}$ define a measurement, since they are positive and sum to the identity, $\sum_{i} W_{i}=\mathbb{1}$. In order to convert $\left|\phi_{d}\right\rangle$ into $|\psi\rangle$, Alice first carries out this measurement, obtaining the outcome $i$ with probability $\left\langle\phi_{d}\left|W_{i}\right| \phi_{d}\right\rangle=1 / d$. She then 
communicates her result to Bob who applies the corresponding unitary operation $\Pi_{i}$, the resulting normalized state being $|\psi\rangle$, as implied by (14).

The quantum-like properties of our local model, i.e., the fact that the hidden variable $|\lambda\rangle$ can be thought of as a quantum state and the quantum form of the response function (6), allow us to adapt Nielsen's construction to it. The idea is that at the source, before sending the classical instructions $|\lambda\rangle$ to each party, a measurement defined by the operators $A_{i}^{*}$ is simulated on $|\lambda\rangle$, giving outcome $i$ with probability $q_{i}(\lambda)=\left\langle\lambda\left|A_{i}^{T} A_{i}^{*}\right| \lambda\right\rangle$. The classical description of the normalized hidden states $\left|\lambda_{i}^{A}\right\rangle=A_{i}^{*}|\lambda\rangle / \sqrt{q_{i}}$ and $\left|\lambda_{i}^{B}\right\rangle=\Pi_{i}|\lambda\rangle$ are then sent, respectively, to Alice and Bob, who use them in the response functions (6) and (7) instead of $|\lambda\rangle$. The joint probabilities $P_{Q R}(a, b)$ predicted by the model for measurements $Q$ and $R$ are thus given by

$$
\begin{aligned}
\int \mu(d \lambda) & \sum_{i=0}^{d-1} q_{i}(\lambda) P_{Q}\left(a \mid \lambda_{i}^{A}\right) P_{R}\left(b \mid \lambda_{i}^{B}\right) \\
= & \sum_{i=0}^{d-1} \int \mu(d \lambda)\left\langle\lambda\left|A_{i}^{T} Q_{a}^{T} A_{i}^{*}\right| \lambda\right\rangle P_{\Pi_{i}^{\dagger} R_{b} \Pi_{i}}(b \mid \lambda)
\end{aligned}
$$

where we used property (8). Replacing the integral in the last expression by the right-hand side of (10), we obtain

$$
\sum_{i=0}^{d-1}\left(\frac{p^{\phi}}{d} \operatorname{tr}\left(A_{i}^{T} Q_{a}^{T} A_{i}^{*} \Pi_{i}^{\dagger} R_{b} \Pi_{i}\right)+\frac{1-p^{\phi}}{d^{2}} \operatorname{tr}\left(A_{i}^{T} Q_{a}^{T} A_{i}^{*}\right)\right) .
$$

Using Eqs. (14) and (5), and the fact that $\sum A_{i} A_{i}^{\dagger}=d \sigma$, where $\sigma=\operatorname{tr}_{B}|\psi\rangle\langle\psi|$, one can check that these probabilities are equal to the quantum probabilities $\operatorname{tr}\left(\tilde{\rho} Q_{a} \otimes R_{b}\right)$ for the state

$$
\tilde{\rho}=p^{\phi}|\psi\rangle\langle\psi|+\left(1-p^{\phi}\right) \sigma \otimes \frac{\mathbb{1}}{d} .
$$

Not surprisingly, the measurement at the source modifies the local noise of Alice, which is no longer completely depolarized, and introduce some bias depending on $|\psi\rangle$.

This result can already be interpreted as a measure of the robustness of the nonlocal correlations of an arbitrary entangled state $|\psi\rangle$. By mixing a state-dependent local noise, with mixing probability $1-p^{\phi}$, it is always possible to wash out the nonlocal correlations of the state $|\psi\rangle$.

In order to extend this result to the case of completely depolarized noise, one can add some extra local noise to Alice such that the resulting state has the form (13), with the penalty that $p<p^{\phi}$. Writing the reduced density matrix $\sigma$ in its diagonal form $\sigma=\sum_{j} \mu_{j}^{2}|j\rangle\langle j|$, and defining $\sigma_{k}=\sum_{j} \mu_{j+k(\bmod d)}^{2}|j\rangle\langle j|$, it is clear that the state

$$
q \tilde{\rho}+\frac{1-q}{d-1} \sum_{k=1}^{d-1} \sigma_{k} \otimes \frac{\mathbb{1}}{d}
$$

has the form (13) for $q\left(1-p_{d}\right)=(1-q) /(d-1)$, in which case the probability $p$ is given by

$$
p^{\rho}=\frac{p^{\phi}}{\left(1-p^{\phi}\right)(d-1)+1} \underset{\text { large } d}{\longrightarrow} \frac{\log (d)}{d^{2}} .
$$

The state (18) is clearly local, since it is a convex combination of local states. We have thus shown that the noisy states (3) have a local model for projective measurements whenever $p \leq p^{\rho}$. The probabilities $p^{\phi}$ and $p^{\rho}$ represent the main results of this work and provide lower bounds on $p_{L}^{\phi}$ and $p_{L}$. Several implications of our findings are discussed in what follows.

First of all, one may ask about the tightness of our bound. Actually, our model is based on Werner's construction, and this model is known not to be tight in the case $d=2[16$. Even if it is not tight, it would be interesting to understand whether the model predicts the right asymptotic dependence with the Hilbert-space dimension $d$. An upper bound on $p_{L}$ follows from the results of [17, where it was shown that a state of the form $\varrho_{2}=$ $p\left|\phi_{2}\right\rangle\left\langle\phi_{2}\right|+(1-p) \mathbb{1} / d^{2}$, where $\left|\phi_{2}\right\rangle=1 / \sqrt{2}(|00\rangle+|11\rangle)$ is a projector onto a two-qubit maximally entangled state, violates the Clauser-Horne-Shimony-Holt inequality [18] whenever $p>p^{\varrho_{2}}$, where

$$
p^{\varrho_{2}}=\frac{4(d-1)}{(\sqrt{2}-1) d^{2}+4 d-4} \underset{\text { large } d}{\longrightarrow} \frac{4}{(\sqrt{2}-1) d},
$$

which tends to zero when $d \rightarrow \infty$. This result together with our previous model thus imply that $p^{\rho} \leq p_{L} \leq p^{\varrho_{2}}$.

Our results, when combined with (20), also provide a strict proof of the fact that the nonlocal correlations of maximally entangled states, under projective measurements, are not the most robust ones. Indeed, we have a local model for isotropic states whenever $p \leq p^{\phi}$, while there exist quantum states of the form (3) violating a Bell inequality when $p>p^{\varrho_{2}}$. For sufficiently large dimension, $p^{\varrho_{2}}<p^{\phi}$, so we have a Bell inequality violation in a range of $p$ for which we have shown the existence of a local model for isotropic states.

It is also interesting to compare the bounds derived here for nonlocality with those known for entanglement. To our knowledge, the best upper and lower bound on the critical probability $p_{S}$ such that the states (3) are guaranteed to be separable were obtained in Ref. [12]:

$$
\frac{1}{d^{2}-1} \leq p_{S} \leq \frac{2}{d^{2}+2} .
$$

Interestingly, the upper bound is obtained, as above, for the case in which the state $\rho$ in (3) is equal to a projector onto $\left|\phi_{2}\right\rangle$. Comparing with Eq. (19), we see that the critical noise probability for nonlocality under projective measurements is, at least, asymptotically $\log (d)$ larger than the one for separability, as it is for isotropic states.

Finally, let us briefly mention how the above results can be extended to the case of general measurements. 
TABLE I: Asymptotic bounds on the critical noise threshold for separability $\left(p_{S}\right)$ and locality $\left(p_{L}\right)$ for maximally entangled states $\left(\left|\phi_{d}\right\rangle\right)$ and arbitrary states $(\rho)$. For maximally entangled states, $p_{S}^{\phi}$ is given in [14]; the lower bounds for $p_{L}^{\phi}$ follow from eqs. (12) and (24); and the upper bounds from [19], where $K$ is Catalan's constant. For arbitrary states, bounds for $p_{S}$ were derived in [12]; the lower bounds for $p_{L}$ are obtained from those for the maximally entangled states using eq. (19); the upper-bounds are those of [17].

\begin{tabular}{c|lll}
\hline \hline state & separability & locality (projective meas.) & locality (general meas.) \\
\hline$\left|\phi_{d}\right\rangle$ & $p_{S}^{\phi}=\frac{1}{d+1}$ & $\Theta\left(\frac{\log d}{d}\right) \leq p_{L}^{\phi} \leq \frac{\pi^{2}}{16 K} \simeq 0.67$ & $\Theta\left(\frac{3}{e d}\right) \leq p_{L}^{\phi} \leq \frac{\pi^{2}}{16 K} \simeq 0.67$ \\
arbitrary $\rho$ & $\frac{1}{d^{2}-1} \leq p_{S} \leq \frac{2}{d^{2}+2}$ & $\Theta\left(\frac{\log d}{d^{2}}\right) \leq p_{L} \leq \Theta\left(\frac{4}{(\sqrt{2}-1) d}\right)$ & $\Theta\left(\frac{3}{e d^{2}}\right) \leq p_{L} \leq \Theta\left(\frac{4}{(\sqrt{2}-1) d}\right)$ \\
\hline \hline
\end{tabular}

The idea is, as above, to start by constructing a model for isotropic states, adapting the one for Werner states of Ref. [4]. As noted in [4], it is sufficient to simulate measurements $M$ and $N$ defined by operators $M_{a}=c_{a} Q_{a}$ and $N_{b}=c_{b} R_{b}$ proportional to one-dimensional projectors $Q_{a}$ and $R_{b}$ to be able to simulate any measurement by Alice and Bob. In our corresponding model, the hidden states are again vectors $|\lambda\rangle$ in $\mathbb{C}^{d}$ chosen with the Haar measure $\mu$. Alice's response function is basically the same as before,

$$
P_{M}(a \mid \lambda)=\left\langle\lambda\left|M_{a}^{T}\right| \lambda\right\rangle,
$$

while Bob's is, taking inspiration from [4], chosen as

$$
\begin{aligned}
P_{N}(b \mid \lambda) & =\left\langle\lambda\left|N_{b}\right| \lambda\right\rangle \Theta\left(\left\langle\lambda\left|R_{b}\right| \lambda\right\rangle-\frac{1}{d}\right) \\
+\frac{c_{b}}{d} & {\left[1-\sum_{k}\left\langle\lambda\left|N_{k}\right| \lambda\right\rangle \Theta\left(\left\langle\lambda\left|R_{k}\right| \lambda\right\rangle-\frac{1}{d}\right)\right], }
\end{aligned}
$$

where $\Theta$ is the Heaviside step function. Evaluation of the integral (2) with the definitions (22) and (23) can be done along the same steps as in [4] and yields the joint measurement outcome probabilities for an isotropic state with the critical value

$$
\tilde{p}^{\phi}=\frac{(3 d-1)(d-1)^{d-1}}{(d+1) d^{d}} \underset{\text { large } d}{\longrightarrow} \frac{3}{e} \frac{1}{d} .
$$

Since this model has the same quantum-like properties as the one for projective measurements, cf. definition (22), it can also be extended to arbitrary noisy states (3) using Nielsen's protocol. The corresponding critical probability is given by (11) with $p^{\phi}$ replaced by the above value of $\tilde{p}^{\phi}$.

In conclusion, we have obtained bounds on the robustness of the nonlocal correlations of arbitrary entangled states. Our results are summarized in Table I. In the particular but interesting case where the state is maximally entangled, we derived better bounds by exploiting the symmetry of isotropic states [13]. Apart from their fundamental significance, our results are interesting from the point of view of the characterization of quantum information resources: if the noise affecting a state is larger than our bounds, its outcome correlations for local measurements can be reproduced by classical means alone.
Note added. While completing this work, we learned that our local model for isotropic states was independently derived in [13] in the context of state steerability. We note that all our models imply the non-steerability of the corresponding quantum states because Alice's response function is always quantum (see [13] for details).

Acknowledgements. We acknowledge financial support from the EU Qubit Applications Project (QAP) Contract number 015848, the Spanish projects FIS2004-05639C02-02, Consolider QOIT, the Spanish MEC for "Ramon y Cajal" and "Juan de la Cierva" grants, the Generalitat de Catalunya, the Fundação para a Ciência e a Tecnologia (Portugal) through the grant SFRH/BD/21915/2005, the National Research Fund of Hungary OTKA under Contract No. T049234, and the Hungarian Academy of Sciences (Bolyai Programme). Research at Perimeter Institute for Theoretical Physics is supported in part by the Government of Canada through NSERC and by the Province of Ontario through MRI.

[1] J. S. Bell, Physics 1, 195 (1964).

[2] N. Gisin, Phys. Lett. A 154, 201 (1991).

[3] R. F. Werner, Phys. Rev. A 40, 4277 (1989).

[4] J. Barrett, Phys. Rev. A 65, 042302 (2002).

[5] G. Tóth and A. Acín, Phys. Rev. A 74, 030306(R) (2006).

[6] R. Jozsa and N. Linden, quant-ph/0201143, G. Vidal, Phys. Rev. Lett. 91, 147902 (2003).

[7] M. Horodecki, P. Horodecki and R. Horodecki, Phys. Rev. A 60, 1888 (1999).

[8] See for instance G. Brassard, quant-ph/0101005, C. Brukner, M. Zukowski, and A. Zeilinger, Phys. Rev. Lett. 89, 197901 (2002).

[9] A. Acín et al. quant-ph/0702152 A. Acín, N. Gisin, and L. Masanes, Phys. Rev. Lett. 97, 120405 (2006); J. Barrett, L. Hardy, and A. Kent, ibid 95, 010503 (2005).

[10] This does not mean that the state can be replaced by shared randomness in any scenario, see Ll. Masanes, Phys. Rev. Lett. 96, 150501 (2006).

[11] K. Zyczkowski, P. Horodecki, A. Sanpera and M. Lewenstein, Phys. Rev. A 58, 883 (1998); G. Vidal and R. Tarrach, Phys. Rev. A 59, 141 (1999); S. L. Braunstein et al., Phys. Rev. Lett. 83, 1054 (1999).

[12] L. Gurvits and H. Barnum, Phys. Rev. A 66, 062311 (2002).

[13] H. M. Wiseman, S. J. Jones and A. C. Doherty, Phys. 
Rev. Lett. 98, 140402 (2007).

[14] M. Horodecki and P. Horodecki, Phys. Rev. A 59, 4206 (1999).

[15] M. A. Nielsen, Phys. Rev. Lett. 83, 436 (1999).

[16] A. Acín, N. Gisin and B. Toner, Phys. Rev. A 73, 062105 (2006).

[17] A. Acín, T. Durt, N. Gisin and J. I. Latorre, Phys. Rev.
A 65, 052325 (2002).

[18] J. F. Clauser, M. A. Horne, A. Shimony and R. A. Holt, Phys. Rev. Lett. 23, 880 (1969).

[19] D. Collins, N. Gisin, N. Linden, S. Massar and S. Popescu, Phys. Rev. Lett. 88, 040404 (2002). 